【Technical Paper】

\title{
Improvement of Flame Retardancy of PET Fabric via UV Induced Grafting of Organic Phosphorus Monomer
}

\author{
Jing Li, Xiao Wang*, \#, Yongzhu Cui, Ruoyuan Song, Lihua Lv, and Chunyan Wei \\ *School of Textile and Material Engineering, Dalian Polytechnic University, Dalian, 116034, China
}

\begin{abstract}
Poly(ethylene terephthalate) (PET) fabric was modified with bis[2-(methacryloyloxy)-ethyl] phosphate using 2,4,6-trimethylbenzoyldiphenyl phosphine oxide as photoinitiator via UV induced grafting to improve flame retardant performance. Effects of monomer concentration, initiator amount, grafting time and ethanol-water volume ratio on grafting yield were investigated. The chemical structure of grafted PET fabric was characterized by FTIR and SEM observation. Thermal behaviour of grafted PET fabric was investigated by thermal gravimetry analysis. The results indicated that the residual char of grafted samples reached $13.6 \%$ at $800^{\circ} \mathrm{C}$, which was higher than that of untreated fabric. The flame retardant bis[2-(methacryloyloxy)-ethyl] phosphate promoted formation of residual char, which improved the anti-dripping property and flame retardancy of PET fabric.
\end{abstract}

(Received April 13, 2016; Accepted July6, 2016)

\section{Introduction}

Poly(ethylene terephthalate) (PET) fabric has been extensively used in both industrial textiles and consumer goods due to a variety of good performances, such as high mechanical strength, excellent resistance to shrinkage, low cost, lowdensity and fairly high mechanical strength etc [1-4]. However, the applications are limited by the combustibility of PET, and the formation of melt dripping during PET fabric burning may result in the spreading of fire [5-7]. Therefore, it is of great necessity to impart both flame retardance and dripping resistance to PET fabric [8-9]. Several flame retardant methods [7-10], such as copolymerizing polyester with flame retardant, melt spinning of a blend of flame retardant and polyester and flameretardant treatment, have been used to enhance the flame retardancy of PET fabric and flame retardant finishing has usually been applied to enhance the flame retardancy of PET fabric owing to its convenience and economic process. Ding's [11] work showed three different monomers such as acrylic acid, acrylamide and dimethyl vinylphosphonate were grafted onto PET fabric respectively by electron beam irradiation method to impart PET either anti- dripping property or flame retardancy. A novel phosphorus-containing flame-retardant copolyester/ montmorillonite nanocomposite (PET-co-HPPPA/OMMT) was synthesized via direct polymerisation of PET monomers, HPPPA dispersed with organically modified montmorillonite (O-MMT) by Ge et al. [12]. D. Q. Chen et al. [13] synthesized a novel anti-dripping flame retardant poly(2-hydroxy propylene spirocyclic pentaerythritol bisphosphonate) (PPPBP) and emloyed dipping-pad-drying-thermo fixation method to finish PET. Compared with above approaches, photo-induced grafting technique has advantages of mild reaction conditions, less damage to the bulk material, long-term stability of the grafted chains and durable flame retardancy [14-17]. In addition, photografting of PET fabric with bis [2(methacryloyloxy)-ethyl] phosphate (DMEP) had rarely been reported for enhancing its anti-dripping property and the grafted PET fabric had thermal behaviour superior to those of the previous researches [18].

In this work, a phosphorous-containing monomer, DMEP, was grafted onto PET fabric using 2,4,6trimethylbenzoyldiphenyl phosphine oxide (TPO) as photoinitiator via UV initiation to improve the flame retardancy and anti-dripping property. The effect of

\# corresponding author: Xiao Wang, wangxiao@dlpu.edu.cn 
initiator amount, monomer concentration and grafting time on graft yield was discussed. The structure, thermal property and flammability of grafted PET fabric were analyzed.

\section{Experimental}

\subsection{Materials}

The plain weave PET fabric $\left(138 \mathrm{~g} / \mathrm{m}^{2}\right)$ was used as received. 2,4,6-trimethylbenzoyldiphenyl phosphine oxide (TPO) was provided by Changsha Xinyu Chemical Co., Ltd.. bis[2-(methacryloyloxy)-ethyl] phosphate (DMEP) was supplied by Sigma-Aldrich, Co.. Ethanol was purchased from Tianjin Kermel chemical reagents Co., Ltd.. All the reagents were analytically pure.

\subsection{UV-induced DMEP grafted polymerization}

Surface graft polymerization of DMEP onto PET fabric initiated by TPO was performed by ultraviolet (UV) initiation. Ultraviolet radiation device (UV, GZJ 100F-XF-2) was supplied by Shanghai Guoda special light source Co., Ltd. Bath ratio of 1:10 was used to prepare DMEP solution with different concentrations by using an ethanol-water mixed solvent. The PET fabric was dipped into DMEP solution at $20^{\circ} \mathrm{C}$ for $30 \mathrm{~min}$, and then padded on a laboratory padder with wet pick up of $120 \%$. Subsequently, the PET fabric was treated under UV irradiation (Fig. 1) and the grafted fabric was rinsed with ethanol and then distilled water, respectively. The monomer concentration and initiator amount were based on the weight of fabric (o.w.f\%) and the weight of monomer (o.w.m.\%), respectively.

\subsection{Characterization}

Scanning electron microscope (SEM, JSM-6460 LV) was used to observe surface morphology of grafted and untreated PET fabrics. Attenuated total reflectance (FTIR, Spectrum One-B) spectrometer were recorded to characterize chemical structure of untreated and grafted PET fabrics. Thermal gravimetry (TG) was performed using a STA PT 1600 thermal analysis instrument at a heating rate of $10^{\circ} \mathrm{C} /$ min from 30 to $800^{\circ} \mathrm{C}$ in nitrogen atmosphere. The fabric strength tester (LAIZHOU ELECTRON INSTRUMENT Co., Ltd, YG 065), (LAIZHOU ELECTRON INSTRUMENT Co., Ltd, YG821L) and Spectrophotometer (Color-Eye 7000 Series) were applied to study the effect of grafting on mechanical properties and color of samples, respectively. The test of mechanical properties of samples in the size of 20 $\mathrm{cm} \times 5 \mathrm{~cm}$ was carried out under the condition of gauge length of $100 \mathrm{~mm}$ and deformation speed of 100

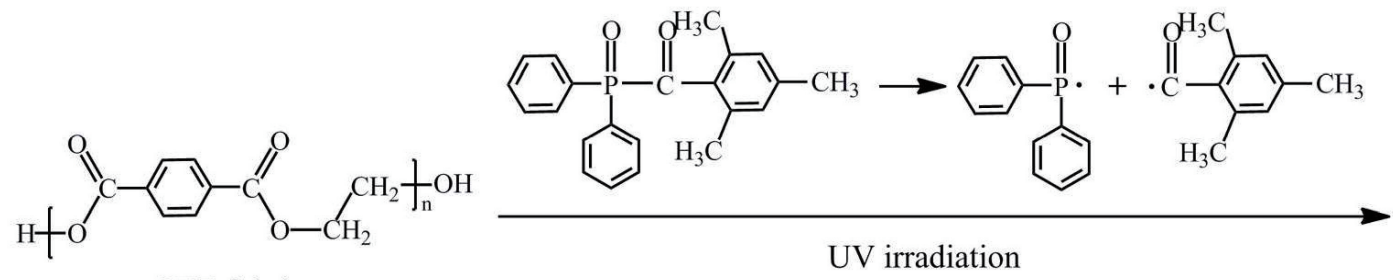

PET fabric<smiles>C=C(C)C(=O)OCCOP(=O)(O)OCCOC(=O)C(=C)C</smiles>

DMEP

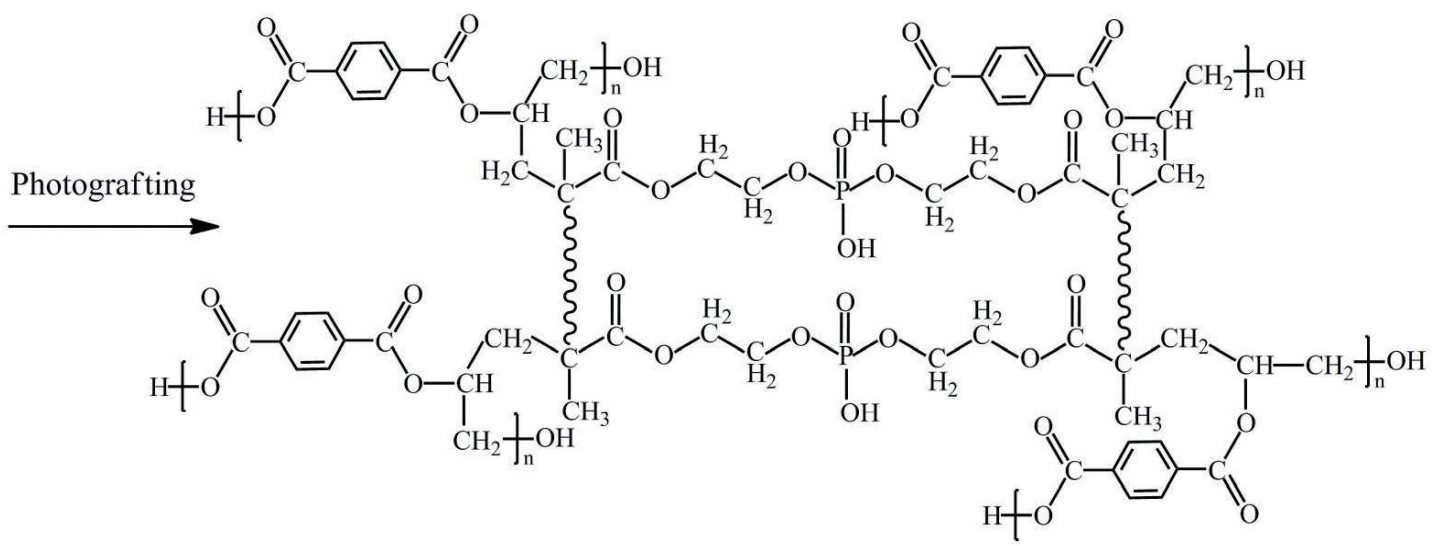

Fig. 1 The chemical mechanism of PET grafted with DMEP. 
$\mathrm{mm} / \mathrm{min}$. The flameproof properties were examined using vertical burning test according to GB/T54552014 (China). During the burning test, the fabric in the size of $30 \mathrm{~cm}$ (height) $\times 8 \mathrm{~cm}$ (width) had been set-up over flame with fixing upper edge and both side edges using some frames and then ignited for $12 \mathrm{~s}$ using a flame of liquefied gas with a height of of $40 \pm 2$ $\mathrm{mm}$. After ignition, after flame time and smoldering time were observed according to the duration time of appearance and disappearance of flame on the fabrics, respectively. The grafting yield was calculated on the basis of the following equation:

$$
\text { Grafting yield }(\%)=\left(\mathrm{W}_{\mathrm{g}}-\mathrm{W}_{0}\right) / \mathrm{W}_{0}
$$

where $\mathrm{W}_{0}$ and $\mathrm{W}_{\mathrm{g}}$ were the weight of PET fabrics before and after grafting, respectively.

\section{Results and discussion}

\subsection{Effect of DMEP concentration on grafting yield}

Fig. 2 displayed the effect of DMEP concentration on grafting yield of PET fabric (3o.w.m.\% of TPO, ethanol-water volume ratio of $1: 9$ and grafting time of $5 \mathrm{~min}$ ). It can be seen that grafting yield increased accordingly with the increasing of DMEP concentration. Graft polymerization predominated at the low concentrations of DMEP below 140 o.w.f\%. Moreover, a high graft yield was obtained at a low concentration, implying good monomer reactivity of DMEP and effective initiation via UV irradiation.

\subsection{Effect of photoinitiator TPO amount on}

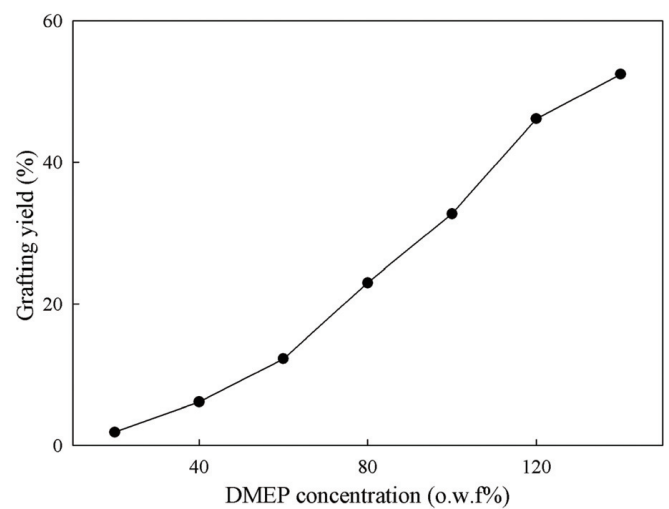

Fig. 2 Effects of DMEP concentration on grafting yield

\section{grafting yield}

The dependence of grafting yield of PET fabric grafted with 100 o.w.f\% of DMEP dissolved in solvent with volume ratio of ethanol-water of $1: 9$ for $5 \mathrm{~min}$ on photoinitiator TPO amount was given in Fig. 3. It was noticed that grafting yield reached a maximum value at 3o.w.m.\% of initiator and further increase of initiator led to decrease of grafting yield. At low concentrations of initiator, more free radicals can be produced with the increasing of initiator amount, resulting in increase of grafting yield. Nevertheless, large amount of radicals generated in a short period of time caused great chances of homopolymerization and decreased grafting yield.

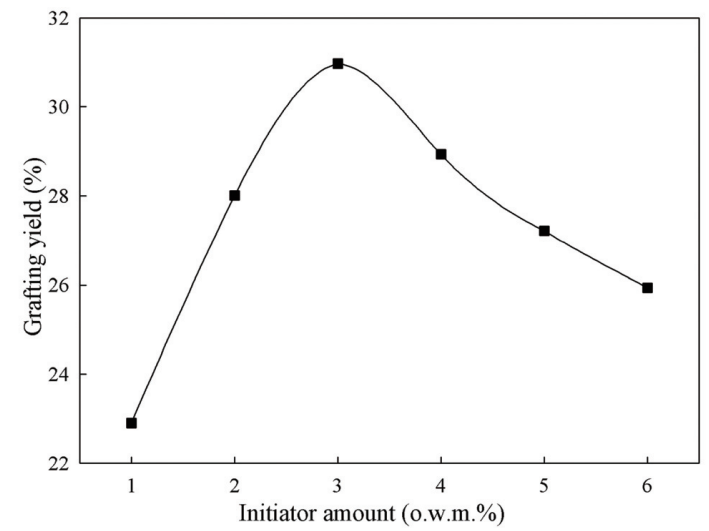

Fig. 3 Effects of initiator amount on grafting yield

\subsection{Effect of grafting time on grafting yield}

As shown in Fig. 4, the grafting yield of PET fabric treated with 100 o.w.f\% of DMEP in solvent with volume ratio of ethanol-water of $1: 9$ initiated by 3 o.w.m.\% of TPO increased rapidly with the increasing amount of grafting time up to $5 \mathrm{~min}$ and no obvious change was found after $5 \mathrm{~min}$. The rising in grafting yield could be attributed to the fact that UV irradiation energy increased with the irradiation time prolonging. More free radicals generated in a prolonged irradiation time were beneficial to initiate graft polymerization. However, too much grafting time destroyed the amorphous region of PET and fabric turned yellow.

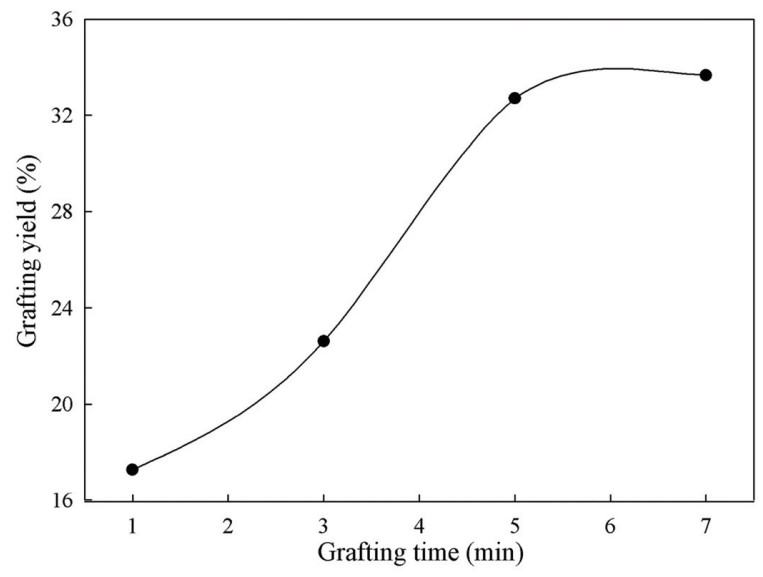

Fig. 4 Effects of grafting time on grafting yield 


\subsection{Effect of ethanol-water volume ratio on grafting yield}

The grafting yield of PET fabric with 100 o.w.f\% of DMEP, 3 o.w.m.\% of TPO and graft time of $5 \mathrm{~min}$ under different ethanol-water volume ratio was listed in Tab. 1. The ethanol-water volume ratio had a large influence on grafting yield. The monomer dissolved well in ethanol, which was in favor of homopolymerization. In addition, ethanol was beneficial to transfer of chain radicals. More free radicals resulted in higher homopolymerization and a low grafting yield at a high amount of ethanol [19].

Table 1 Analysis data of grafting yield and ethanolwater volume ratio

\begin{tabular}{cc}
\hline ethanol-water volume ratio & grafting yield (\%) \\
\hline $1: 9$ & 28.33 \\
$2: 8$ & 26.79 \\
$3: 7$ & 11.4 \\
$4: 6$ & 1.45 \\
\hline
\end{tabular}

\subsection{Flame retardant performance}

The flame retardant performance of PET fabric under 3 o.w.m.\% of TPO, ethanol-water volume ratio of $1: 9$ and graft time of 5 min was tested. It was seen from Tab. 2 that melt dripping disappeared at the monomer concentrations higher than 60 o.w.f\%. When grafting yield was lower than $12.46 \%$, the grafted retardant polymer was not enough to prevent melt dripping. Nevertheless, combining the retardant effect and melt dripping, PET fabric was inclined to cease burning. The damage area also decreased. At a medium grafting yield of $12.46 \%$, better flame retardant effect of grafted PET was attributed to the condensed phase mechanism by changing the pyrolytic path of PET fabric, promoting the carbonization of PET molecules and reducing the generation of combustibles. However, the further increase of grafting yield could facilitate polymerization and carbonization of DMEP and initiate "skeleton effect", resulting in flame spreading and hence increase of after flame time and damage

Table 2 Flame retardant properties of grafted PET fabrics

\begin{tabular}{cccccc}
\hline $\begin{array}{c}\text { monomer } \\
\text { concentration } \\
\text { (o.w.f\%) }\end{array}$ & $\begin{array}{c}\text { grafting } \\
\text { yield (\%) }\end{array}$ & $\begin{array}{c}\text { after } \\
\text { flame } \\
\text { time (s) }\end{array}$ & $\begin{array}{c}\text { smoldering } \\
\text { time (s) }\end{array}$ & $\begin{array}{c}\text { damage } \\
\text { length } \\
(\mathrm{mm})\end{array}$ & $\begin{array}{c}\text { melt } \\
\text { dripping }\end{array}$ \\
\hline 0 & 0 & 36.5 & 0 & 280 & yes \\
20 & 1.9 & 5 & 0 & 230 & yes \\
40 & 6.15 & 8 & 0 & 185 & yes \\
60 & 12.46 & 5 & 0 & 115 & no \\
80 & 20.39 & 12.5 & 0 & 125 & no \\
100 & 27.78 & 16.3 & 0 & 180 & no \\
120 & 37.52 & 40 & 0 & 320 & no \\
\hline
\end{tabular}

length.

\subsection{Effect of grafting on mechanical properties}

It can be learnt from the Tab. 3 that the strength of grafted PET fabric with grafting yield of $12.46 \%$ decreased in a certain degree and the rigidity performed the opposite trend compared with pristine PET. The results may be due to the decrease of space and relative motion between fibers and yarns and hence increase in rigidity of PET fabric, resulting from the polymerization of DMEP grafted onto the surface of PET fabric. According to instrument, breaking time, rigidity and rigidity index refer to the time needed for fabric from beginning loading to rupture, the ratio of difference between bending resistance and midpoint displacement and the ratio of rigidity and fabric thickness, respectively.

Table 3 The physical properties of untreated and grafted PET fabrics

\begin{tabular}{ccc}
\hline performance index & untreated PET & grafted PET \\
\hline breaking strength(N) & 1917 & 1766 \\
elongation at break(\%) & 38.83 & 34.2 \\
work of fracture $(\mathrm{J})$ & 37.4 & 29.0 \\
breaking time $(\mathrm{s})$ & 23.32 & 20.54 \\
bending rigidity $(\mathrm{cN} / \mathrm{mm})$ & 4.09 & 7.18 \\
rigidity index $\left(\mathrm{cN} / \mathrm{mm}^{2}\right)$ & 0.20 & 0.36 \\
\hline
\end{tabular}

\subsection{Effect of grafting on color}

The effect of grafting on color of fabrics was shown in Tab.4. The little difference between $\mathrm{K} / \mathrm{S}$ values (dyeing deepness) of untreated and grafted PET fabrics and small changes in DL* (difference in lightness/darkness value), $\mathrm{Da}^{*}$ (difference on red/ green axis) and $\mathrm{Db}^{*}$ (difference on yellow/blue axis) and $\mathrm{DE}^{*}$ (total color difference value) values implied that the effect of grafting on color of fabrics was slight and negligible. Thus, the appearance of grafted PET fabric was not affected by grafting of DMEP.

Table 4 The color values of untreated and grafted PET fabrics

\begin{tabular}{cccccc}
\hline samples & $\mathrm{K} / \mathrm{S}$ & $\mathrm{DL}^{*}$ & $\mathrm{Du}^{*}$ & $\mathrm{Db}^{*}$ & $\mathrm{DE}^{*}$ \\
\hline $\begin{array}{c}\text { untreated PET } \\
\text { grafted PET }\end{array}$ & 2.81 & 0.02 & 0.33 & 0.41 & 0.53 \\
\hline
\end{tabular}

\subsection{FTIR analysis}

FTIR spectra of untreated PET fabric and grafted PET fabric with grafting yield of $12.46 \%$ were shown in Fig. 5. In the spectrum of untreated PET fabric, characteristic absorption bands at $3429 \mathrm{~cm}^{-1}$ was attributed to the stretching vibration of $\mathrm{O}-\mathrm{H}$ group. The absorption peak of $-\mathrm{CH}_{2}$ appeared at $2970 \mathrm{~cm}^{-1}$. The appearance of $-\mathrm{C}=\mathrm{O}$ absorption peak was found at $1717 \mathrm{~cm}^{-1}$. In comparison with untreated PET fabric, the typical absorption bands of PET 
fabric were found in the spectrum of grafted sample. The new absorption peak of $\mathrm{O}-\mathrm{P}=\mathrm{O}$ at $1197 \mathrm{~cm}^{-1}$ indicated that DMEP was grafted onto the surface of PET fabric.

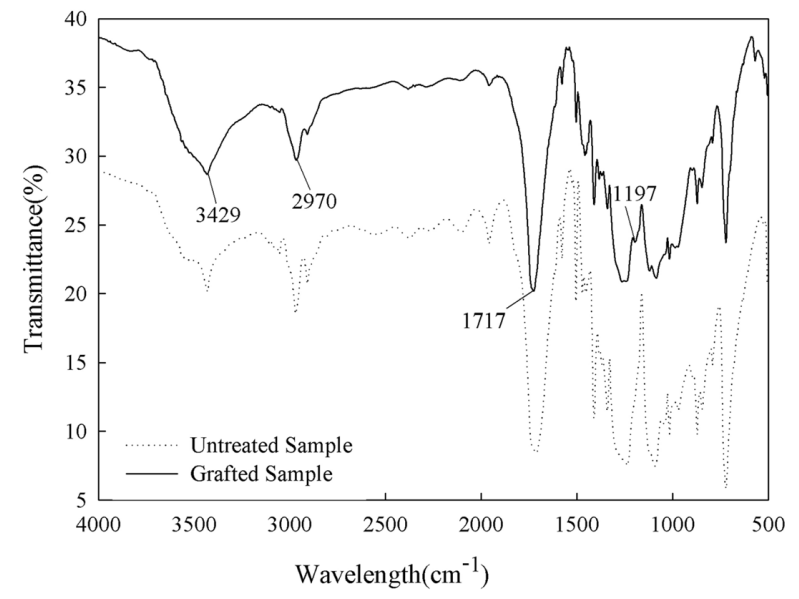

Fig. 5 FTIR spectra of untreated and grafted PET fabrics

\subsection{Surface morphology}

SEM micrographs of untreated PET fabric and grafted PET fabric with grafting yield of $12.46 \%$ were shown in Fig. 6. The surface morphology of grafted PET fabric sample was significantly influenced by graft polymerization. The surface of untreated PET fabric was smooth, while the surface of grafted fabric was rough and entirely covered with granule-like graft polymers.
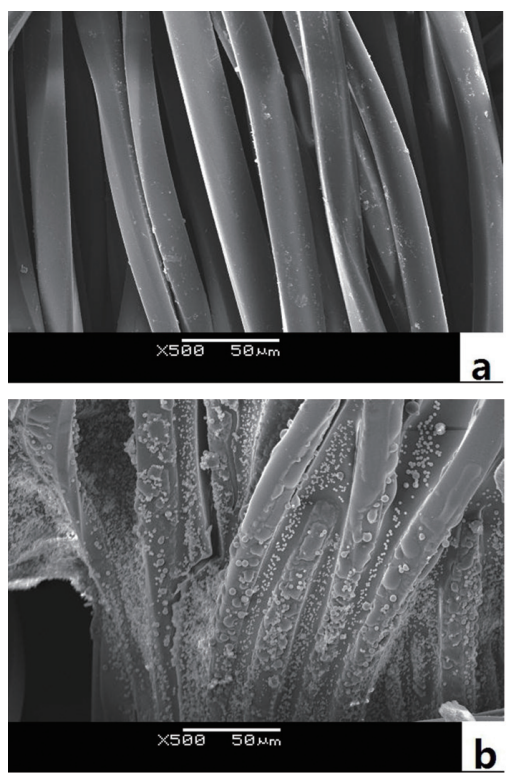

Fig. 6 SEM images of PET fabrics: (a) untreated fabric, (b) grafted fabric

\subsection{TG analysis}

Fig. 7 showed TG curves of untreated and grafted PET fabrics with grafting yield of $12.46 \%$. Untreated PET fabric started decomposing at about $375^{\circ} \mathrm{C}$, while grafted PET fabric started at $295^{\circ} \mathrm{C}$. The initial thermal degradation of grafted PET fabric in the first thermal decomposition was mainly attributed to the phosphoric acid catalyzed. The char yield of grafted PET fabric of $13.6 \%$ at $800^{\circ} \mathrm{C}$ was higher than that of untreated PET of $5.4 \%$. This implied that the carbonization of PET molecules was promoted by the grafted DMEP polymers which changed the pyrolytic path and promoted the condensation, cyclization and crosslinking of PET molecules.

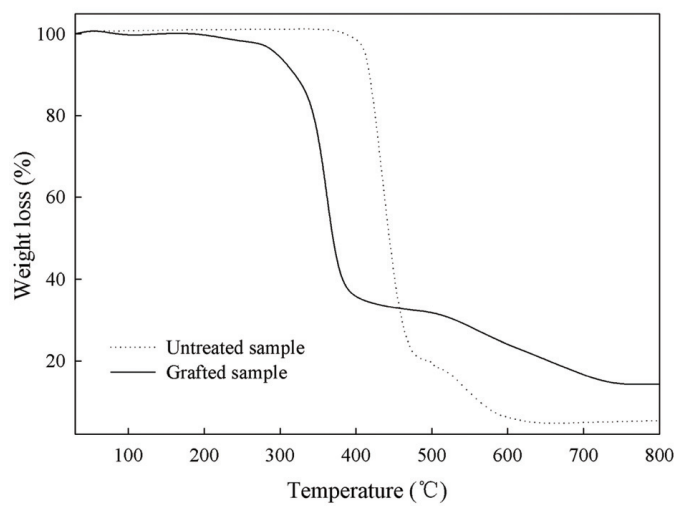

Fig. 7 TG curves of PET fabric before and after grafting

\section{Conclusion}

High graft yield of DMEP monomer onto PET fabric was achieved under the conditions of photoinitiator amount of 3 o.w.m.\%, grafting time of 5 min and ethanol-water volume ratio of $1: 9$. The phenomenon of melt dripping disappeared at the DMEP concentrations higher than 60 o.w.f\%. The after flame time decreased to $5 \mathrm{~s}$ at a medium graft yield of $12.46 \%$. Grafting of DMEP improved carbonization of PET molecules, anti-dripping and flameproof performance of PET fabric.

\section{Acknowledgement}

This work was financially supported by Cultivation Project of Youth Excellence of Dalian, Liaoning Province P. R. China (Project No. 2015R 082).

\section{References}

1. S. V. Levchik, and E.D. Weil, Polymer International, 4, 11 - 35 (2005).

2. S.-C. Yang, and J. P. Kim, Journal of Applied Polymer Science, 108, 2297 - 2300 (2008).

3. F. Xu, F. X. Zhang, G. X. Zhang, and Y. S. Zhang, 
Advanced Materials Research, 1004-1005, 315-318 (2014).

4. J. N. Wang, X. Y. Su, and Z. P. Mao, Polymer Degradation and Stability, 109, 154-161 (2014) .

5. Y. Z. Wang, X. T. Chen, X. D. Tang, and X. H. Du, Journal of Materials Chemistry, 13, 1248-1249 (2003).

6. D. M. Ban, Y. Z. Wang, B. Yang, and G. M. Zhao, European Polymer Journal, 40, 1909-1913 (2004).

7. S. Y. Liang, N. M. Neisius, and S. Gaan, Progress in Organic Coatings, 76, 1642-1665 (2013).

8. K. Opwis, A. Wego, T. Bahners, and E. Schollmeyer, Polymer Degradation and Stability, 96, 393-395 (2011).

9. Y.-H. Kim, J. Jang, K.-G. Song, E.-S. Lee, and S.-W. Ko, Journal of Applied Polymer Science, 81, 793-799 (2001).

10. A. R. Horrocks, Polymer Degradation and Stability, 96, 377 - 392 (2011).

11. C. Ding, J. C. Zhang, X. Q. Liu, X. X. Feng, H. Zhang, and K. Lai, Fibers and Polymers, 15, 18951901 (2014).
12. X. G. Ge, D. Y. Wang, C. Wang, M. H. Qu, J. S. Wang, C. S. Zhao, X. K. Jing, and Y. Z. Wang, European Polymer Journal, 43, 2882-2890 (2007).

13. D. Q. Chen, Y. Z. Wang, X. P. Hu, D. Y. Wang, M. H. Qu, and B. Yang, Polymer Degradation and Stability, 88, 349 - 356 (2005).

14. J. P. Deng, L. F. Wang, L. Y. Liu, and W. T. Yang, Progress in Polymer Science, 34, 156-193 (2009).

15. L. H. Yu, S. Zhang, W. Liu, X. J. Zhu, X. P. Chen, and X. S. Chen, Polymer Degradation and Stability, 95, 1934-1942 (2010).

16. Q. L. Feng, X. Y. Gu, S. Zhang, B. Zhao, J. Sun, X. Y. Li, and M. Z. Dong, Industrial and Engineering Chemistry Research, 51, 14708-14713 (2012).

17. S. L. Gao, R. Häßler, E. Mäder, T. Bahners, K. Opwis, and E. Schollmeyer, Applied Physics B : Lasers and Optics, 81, 681 - 690 (2005).

18. J. Jang, and Y.-K. Jeong, Fibers and Polymers, 9, $667-673$ (2008).

19. K. E. Russell, Progress in Polymer Science, 27, 1007 1038 (2002). 